\title{
Intraoperative transprosthetic leakage of a bovine prosthetic valve after aortic valve replacement
}

\author{
Mamoru Arakawa, MD, PhD, Kazunori Hashimoto, MD, and Homare Okamura, MD, PhD, Tokyo, Japan
}

\author{
From the Department of Cardiovascular Surgery, Nerima Hikarigaoka Hospital, Tokyo, Japan. \\ Disclosures: Authors have nothing to disclose with regard to commercial support. \\ Received for publication March 5, 2017; revisions received April 8, 2017; accepted for publication April 22, 2017; \\ available ahead of print June 24, 2017. \\ Address for reprints: Mamoru Arakawa, MD, PhD, 2-11-1 Hikarigaoka, Nerima-Ku, Tokyo 179-0072, Japan \\ (E-mail: a_mamoru@mbn.nifty.com). \\ J Thorac Cardiovasc Surg 2017;154:e35-6 \\ $0022-5223 / \$ 36.00$ \\ Copyright (c) 2017 by The American Association for Thoracic Surgery \\ http://dx.doi.org/10.1016/j.jtcvs.2017.04.080
}

\section{PTCS
VIDEO
CIIP...}

Video clip is available online.

In cases of intraoperative aortic regurgitation after aortic valve replacement, transvalvular and paravalvular leakage are usually considered as differential diagnoses. We report a rare case of intraoperative transprosthetic leakage of a Carpentier Edwards Perimount (CEP) Magna bioprosthesis (Edwards Lifesciences, Irvine, Calif).

\section{CLINICAL SUMMARY}

An 88-year-old man presented with dyspnea on excursion and was referred to our institution. His past medical history included renal failure necessitating hemodialysis. Transthoracic echocardiography demonstrated severe aortic valve regurgitation and moderate mitral regurgitation caused by mitral annulus dilation. The left ventricular ejection fraction was $53 \%$, and left ventricular diastolic dimension was $67 \mathrm{~mm}$. Electrocardiography showed sinus rhythm and coronary angiography revealed no stenosis.

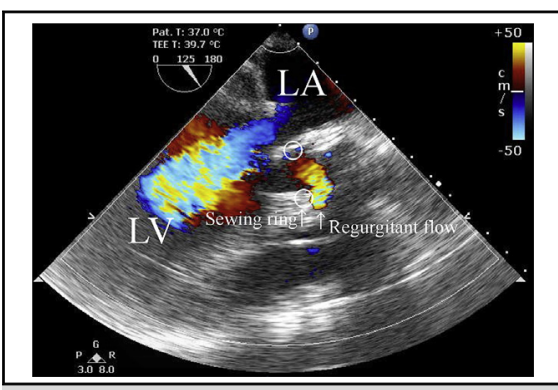

Regurgitant flow from just above the sewing ring to the anterior mitral leaflet.

\section{Central Message}

Transprosthetic leakage, rather than transvalvular or paravalvular leakage, needs to be included in the differential diagnosis in cases of aortic regurgitation after bovine prosthetic valve replacement.

See Editorial Commentary page e37.
Aortic valve replacement and mitral valve repair were performed via median sternotomy. Cardiopulmonary bypass was established by cannulation of the ascending aorta, superior vena cava, and inferior vena cava. A left ventricular vent was inserted. Antegrade and retrograde cold blood cardioplegia were administered intermittently. Mitral regurgitation was repaired by annuloplasty with a $30-\mathrm{mm}$ Carpentier Edwards Physio II ring (Edwards Lifesciences). The aortic valve was tricuspid, and the noncoronary cusp was prolapsed into the left ventricle.
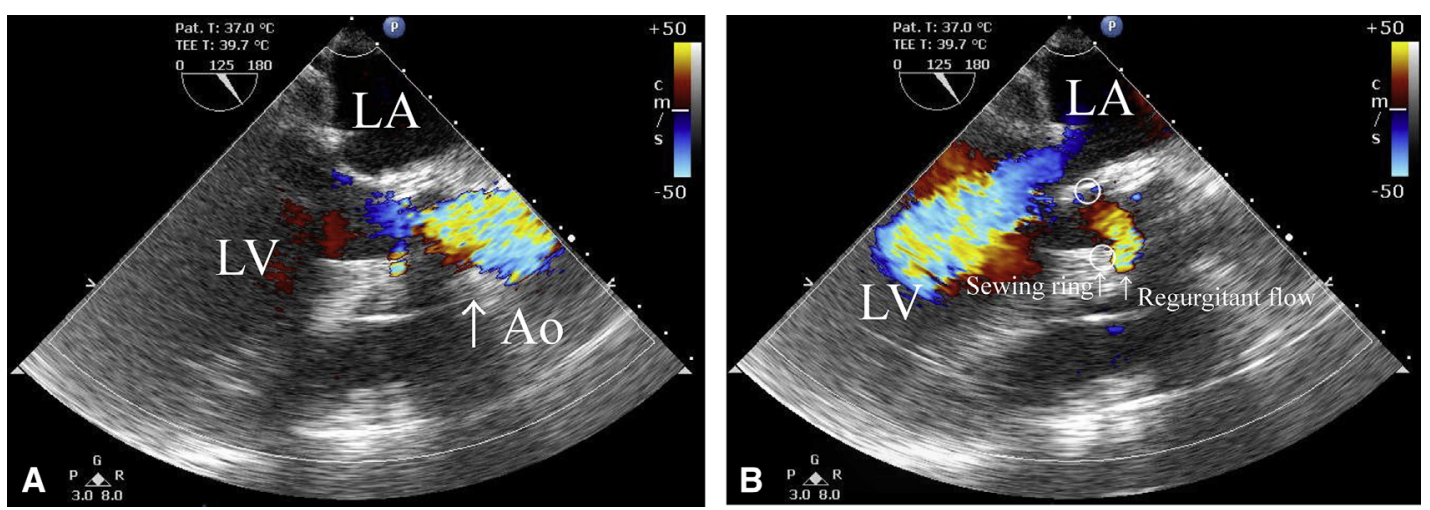

FIGURE 1. Long-axis view of intraoperative transesophageal echocardiography in diastolic phase without (A) and with (B) color Doppler imaging. $L V$, Left ventricle; $L A$, left atrium; $A o$, ascending aorta. 


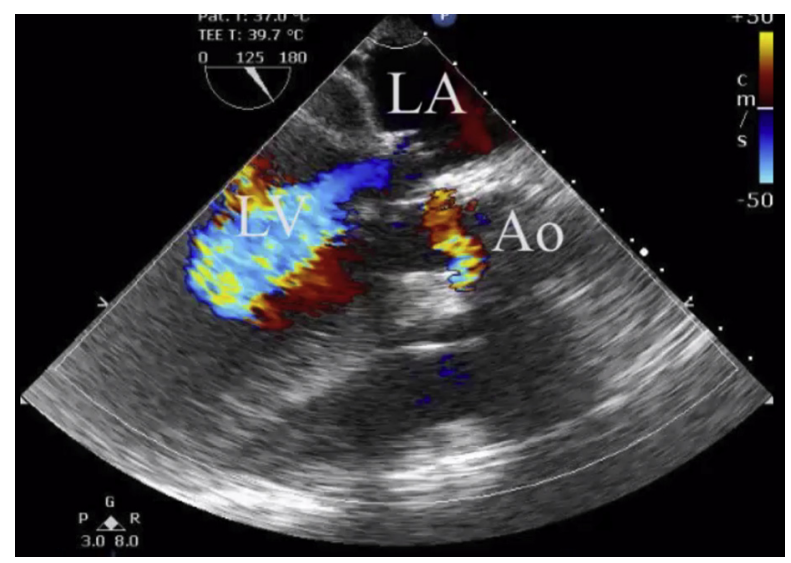

VIDEO 1. Long- and short-axis view of intraoperative transesophageal echocardiography. $L A$, Left atrium; Ao, ascending aorta. Video available at: http://www.jtcvsonline.org/article/S0022-5223(17)31102-9/addons.

Once resection of the aortic valve was completed, a 21-mm CEP Magna aortic heart valve was implanted in the supra-annular position using 2-0 polyester noneverting mattress sutures with pledgets. After closure of the aortotomy and declamping, transesophageal echocardiography (TEE) showed leakage through the prosthesis in the aortic position (Figure 1 and Video 1). The leakage flowed from the original right coronary cusp position to the anterior mitral leaflet, passing between the sewing ring of the stented valve and stent post. Because the flow route of the leakage was distal to the sewing cuff obviously, we excluded the possibility of paravaluvular leakage (Figures 1 and 2). Even after the pump was completely off, transprosthetic leakage continued, without hemodynamic instability. We considered the cause of the leakage to be valve dysfunction, such as a tear in the valve leaflet or a fixed leaflet in the open position. We resumed the pump, clamped the aorta, and inspected the implanted valve. No possible causes of paravaluvular leakage, such as a tear at the aortic annulus or a gap in the suture line, were found.

The initially implanted bioprosthesis was excised, and another CEP Magna valve of the same size was reimplanted. The removed prosthesis appeared to be intact, with no tears or abnormal movement of the leaflets. The patient was uneventfully weaned from the cardiopulmonary pump. He was discharged 14 days after surgery without any complications.

The excised valve was sent to the manufacturer for a complete examination. The manufacturer reported no abnormalities in the valve, including the leaflets, the sewing cuff, the stent post, or the fabric.

\section{DISCUSSION}

The CEP Magna valve is one of the most frequently implanted tissue valves worldwide. We report a rare

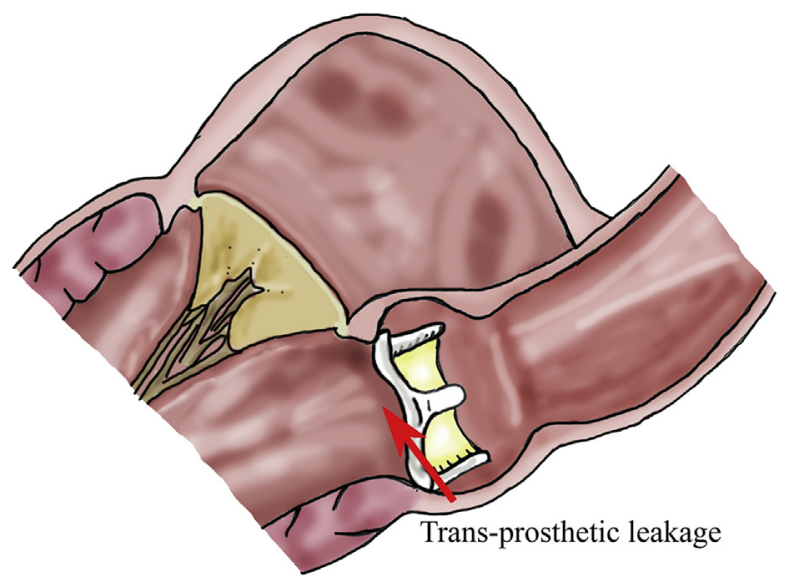

FIGURE 2. Schematic image showing transprosthetic leakage after aortic valve replacement. The transprosthetic leakage is located between the stent post and the sewing ring.

transprosthetic leakage diagnosed by intraoperative TEE. In our case, the explanted prosthetic valve was apparently normal, as confirmed by a detailed examination. According to the type of regurgitant flow observed in the intraoperative TEE, the cause of leakage in the aortic position was considered transprosthetic leakage, that is, neither transvalvular nor paravalvular leakage.

Central transvalvular leakage and paravalvular leakage are the 2 major types of leakage after valve replacement; however, transprosthetic leakage also needs to be considered as another possible cause of leakage. ${ }^{1,2}$ Kuroda and colleagues reported a similar case of regurgitant flow originating from the base of the stent post toward the anterior mitral valve immediately after aortic valve replacement. ${ }^{3}$ The leakage was between the stent post and the sewing ring. In their case, protamine was administered, and the leakage essentially disappeared. ${ }^{3}$

In conclusion, we need to consider transprosthetic leakage as a possible differential diagnoses in patients with intraoperative aortic regurgitation after aortic valve replacement. The accurate diagnosis of transprosthetic leakage may help surgeons choose an approach that can avoid unnecessary reimplantation of a prosthesis.

\section{References}

1. Kunisawa T, Sasakawa T, Kishi M, Suzuki A, Takahata O, Iwasaki H. Transvalvular leakage after the implantation of stented bovine pericardial valves is not only central. J Anesth. 2009;23:639-40.

2. Kunisawa T, Takahashi K, Suzuki A, Takahata O, Iwasaki H. Mild nonpara-/ nontransvalvular leakage of a stented porcine valve implanted in the mitral position decreased after the administration of protamine and disappeared after surgery. J Cardiothorac Vasc Anesth. 2008;22:799-800.

3. Kuroda M, Sudo T, Koizuka S, Nishikawa K, Kadoi Y, Saito S. Regurgitant leak from the area between the stent post and the sewing ring of a stented bovine pericardial valve implanted in the aortic valve position. Cardiovasc Ultrasound. 2010; 8:52. 